\title{
Visceral obesity is not an independent risk factor of mortality in subjects over 65 years
}

This article was published in the following Dove Press journal:

Vascular Health and Risk Management

21 November 2013

Number of times this article has been viewed

\section{Frédérique Thomas' \\ Bruno Pannier' \\ Athanase Benetos ${ }^{2}$ \\ Ulrich MVischer ${ }^{3, \dagger}$ \\ 'Centre d'Investigations Préventives et Cliniques, Paris, France; \\ ${ }^{2}$ Department of Geriatrics, Nancy University Hospital Center, University of Lorraine, Nancy, France; ${ }^{3}$ Geneva University Hospitals, Department of Rehabilitation and Geriatrics, Geneva, Switzerland}

tUlrich MVischer passed away on March 19, 2012
Correspondence: Frédérique Thomas Centre d'Investigations Préventives et Cliniques, 6 rue La Pérouse,

75116 Paris, France

Tel +33 I536735I9

Email thomas@ipc.asso.fr
Abstract: The aim of the study was to determine the role of obesity evaluated by body mass index (BMI), waist circumference (WC), and their combined effect on all-cause mortality according to age and related risk factors. This study included 119,090 subjects (79,325 men and 39,765 women), aged from 17 years to 85 years, who had a general health checkup at the Centre d'Investigations Préventives et Cliniques, Paris, France. The mean follow-up was $5.6 \pm 2.4$ years. The prevalence of obesity, defined by WC and BMI categories, was determined according to age groups ( $<55,55-65,>65$ years). All-cause mortality according to obesity and age was determined using Cox regression analysis, adjusted for related risk factors and previous cardiovascular events. For the entire population, WC adjusted for BMI, an index of central obesity, was strongly associated with mortality, even after adjustment for hypertension, dyslipidemia, and diabetes. The prevalence of obesity increased with age, notably when defined by WC. Nonetheless, the association between WC adjusted for BMI and mortality was not observed in subjects $>65$ years old (hazard ratio $[\mathrm{HR}]=1.010, P=\mathrm{NS})$ but was found in subjects $<55(\mathrm{HR}=1.030, P<0.0001)$ and 55-65 years old $(\mathrm{HR}=1.023, P<0.05)$. By contrast, hypertension $(\mathrm{HR}=1.31, P<0.05)$, previous cardiovascular events $(\mathrm{HR}=1.98, P<0.05)$, and smoking $(\mathrm{HR}=1.33, P<0.05)$ remained associated with mortality even after age 65 . In conclusion, WC adjusted for BMI is strongly and independently associated with all-cause mortality before 65 years of age, after taking into account the associated risk factors. This relationship disappears in subjects $>65$ years of age, suggesting a differential impact of visceral fat deposition according to age.

Keywords: abdominal, aging, body mass index, hypertension, smoking

\section{Introduction}

The association between obesity and cardiovascular (CV) all-cause mortality is well established and is attributed to the negative impact of excess body fat. However, there is still discussion about visceral obesity and its impact on mortality, and especially the effect of this relationship among the elderly. Large collaborative studies using body mass index (BMI) to define obesity have shown that the mortality risk associated with obesity markedly declines with age. ${ }^{1,2}$ By contrast, the risk persists, even in the highest age categories, in studies using waist circumference (WC) to define obesity. ${ }^{3,4}$ However, we have shown in a large population that WC and metabolic syndrome have limited prognostic value in older patients, confirming smaller studies. ${ }^{5,6,7}$ These discrepant results may be accounted for by the limitations of the criteria used to define obesity in clinical practice. BMI is based on body weight and height only and does not directly reflect adiposity. WC is strongly related to central adiposity ${ }^{8}$ and is associated with $\mathrm{CV}$ risk factors, such as hypertension, dyslipidemia, and glucose intolerance. ${ }^{9}$ 
The physiopathological links include inflammation, excessive oxidative stress, and insulin resistance - all deleterious phenomena. While they are quite strongly associated with each other, particularly in individuals with high body corpulence, ${ }^{10} \mathrm{WC}$ and BMI appear to measure distinct features of body composition. For a given BMI value, the WC reflects the abdominal fat deposits. Conversely, for a given WC, the BMI probably reflects non-abdominal as well as abdominal fat deposits and possibly fat-free mass. ${ }^{11}$ The discrepancy between these two measures is likely to increase with advancing age, compounding the assessment of the impact of aging on the prognostic significance of obesity. BMI-adjusted WC may be a better marker of central adiposity than WC alone. ${ }^{11}$ Another important element is the duration of exposure to these risk factors. Older patients with recent obesity, hypertension, dyslipidemia, and diabetes may not be threatened with the same mortality risk as patients who have presented these risk factors for many years or decades. Metabolic syndrome was previously studied in our population (but was not assessed in this study). ${ }^{5}$ Finally, the absolute mortality risk associated with obesity may result in a significantly elevated relative risk in young persons, but not in older persons, for whom a higher all-cause mortality rate is due to age per se or to comorbidities.

The aim of the study was to determine the role of obesity evaluated by BMI, WC, and their combined effect on allcause mortality according to age and related risk factors, in a large French population.

\section{Methods and procedures}

Participants were examined at the Centre d'Investigations Préventives et Cliniques (IPC Center), Paris, France. This medical center, which is subsidized by the French national health care system, offers all working and retired individuals and their families a free medical examination every 5 years. It is one of the largest medical centers of this kind in France, carrying out $\sim 25,000$ health examinations per year for people living in the Paris area. The IPC Center received authorization from the Comité National d'Informatique et des Libertés to conduct and publish these analyses. All subjects gave informed consent at the time of the examination.

The study population, consisting of 129,655 subjects, has been described previously. ${ }^{5}$ In a preliminary analysis, it was found that the association between mortality and obesity was weaker when patients with a low BMI or a short follow-up were included.

Because subjects with very low BMI or subjects who died during the first 2 years of follow-up (representing $8.1 \%$ of our population) had a large probability of having previously undiagnosed pathologies, which could modify the relation between obesity and mortality, the present analysis involved 119,090 subjects, aged from 17 years to 85 years, who had a BMI $>20 \mathrm{~kg} / \mathrm{m}^{2}$ and a follow-up of at least 2 years. The patients were evaluated between January 1999 and December 2006. Follow-up for all-cause mortality was obtained until December 2008. The mean follow-up was 5.6 \pm 2.4 years. During this period, 1,259 subjects died (954 men and 305 women).

\section{Data collection, laboratory measurements, and follow-up}

WC was measured in the standing position, using a tape placed midway between the lower ribs and iliac crests on the midaxillary line. BMI $\left(\mathrm{kg} / \mathrm{m}^{2}\right)$ was calculated from the measured height and body weight. Supine blood pressure was measured in the right arm using a manual mercury sphygmomanometer, after a 10-minute rest. The first and the fifth Korotkoff phases were used to define the systolic blood pressure (BP) and diastolic BP. Three measurements were taken, and the mean of the last two was used. Standard biological parameters were measured by enzymatic methods using a Hitachi 917 automated analyzer (Hitachi Ltd, Tokyo, Japan); High-density lipoprotein (HDL) cholesterol was measured by direct enzymatic assay using cyclodextrin and lowdensity lipoprotein (LDL) cholesterol was calculated using the Friedwald formula. A resting electrocardiogram (EKG) was recorded. A cardiologist encoded EKG abnormalities as rhythmic disorders, QRS complex abnormalities, or depolarization disturbance. Tobacco consumption (never smoker, ex-smoker, or current smoker), physical activity ( $>2$ hours regular physical activity per week), personal medical history, current medication, and alcohol consumption were assessed using a self-administered questionnaire. White-collar workers were categorized as "high socioprofessionals." All clinical and biological parameters were evaluated at a single visit. For each screened subject, vital status was obtained from the French National Institute of Statistics and Economic Studies (Institut National de Statistiques et d'Etudes Economiques), as previously validated and described. ${ }^{12}$

\section{Statistics}

Patient characteristics were compared according to age groups $(<55,55-65$ years, $>65$ years $)$ using analysis of variance (ANOVA) for quantitative parameters, and chi-square tests for dichotomous variables. The distribution of subjects and percentage of deaths according to BMI categories (20-24.9 kg/ $\left.\mathrm{m}^{2}, 25-29.9 \mathrm{~kg} / \mathrm{m}^{2}, \geq 30 \mathrm{~kg} / \mathrm{m}^{2}\right)$ and $\mathrm{WC}$ categories $(<94 \mathrm{~cm}$, 
94-101 cm, $\geq 102 \mathrm{~cm}$, in men; and $<80 \mathrm{~cm}, 80-87 \mathrm{~cm}$, $\geq 88 \mathrm{~cm}$, in women) were analyzed using the chi-square test. The risk of all-cause mortality associated with BMI $\left(\mathrm{kg} / \mathrm{m}^{2}\right)$ and WC $(\mathrm{cm})$ was further evaluated using Cox regression analysis. Hazard ratios (HRs) were calculated per one unit of each parameter. The effect of age on these associations was evaluated again after dividing the population into the three age groups. The analysis was first performed including age, sex, previous CV events, EKG abnormalities, current smoking, socioprofessional category, physical activity, and family history of diabetes and CV disease. An adjustment for hypertension, lipid level, and diabetes, which reflect possible mechanisms by which obesity increases mortality, was made in separate, subsequent steps. For these analyses, hypertension was defined as systolic BP $>140 \mathrm{mmHg}$, diastolic BP $>90 \mathrm{mmHg}$, or ongoing antihypertensive treatment, and diabetes was defined as a fasting serum glucose $>126 \mathrm{mg} / \mathrm{dL}$ or ongoing treatment. Current smokers were compared with the pooled subgroups of nonsmokers and former smokers. Previous CV events represented previous myocardial infarction or stroke, by self-report. For obesity markers, different models were used, and BMI and WC were further adjusted for each other (WC adjusted for BMI and BMI adjusted for WC).

All statistical analyses, including interactions, were performed using SAS 8.2 statistical software (SAS Institute Inc, Cary, NC, USA).

\section{Results \\ Population characteristics}

The study participant characteristics according to age groups ( $<55$ years, 55-65 years, $>65$ years) and sex are shown in Table 1 . The prevalence of elevated WC $(\geq 88 \mathrm{~cm}$ in women, $\geq 102 \mathrm{~cm}$ in men) markedly increased with age, whereas the increase in elevated BMI $\left(\geq 30 \mathrm{~kg} / \mathrm{m}^{2}\right)$

Table I Population characteristics (mean \pm SEM for quantitative variable and $\%[\mathrm{n}]$ for dichotomous variables), according to age groups

\begin{tabular}{|c|c|c|c|c|c|c|c|c|}
\hline & \multicolumn{4}{|l|}{ Men } & \multicolumn{4}{|l|}{ Women } \\
\hline & $<\mathbf{5 5}$ years & $55-65$ years & $>65$ years & $P$-value & $<55$ years & $55-65$ years & $>65$ years & $P$-value \\
\hline $\mathrm{N}$ & 63,365 & 12,876 & 3,084 & & 28,649 & 7,799 & 3,317 & \\
\hline Age (years) & $40.7 \pm 8.7$ & $58.5 \pm 2.7$ & $68.9 \pm 3.3$ & & $39.3(10.1)$ & $58.9(2.7)$ & $69.3(3.3)$ & \\
\hline Weight (kg) & $78.2 \pm 11.8$ & $78.9 \pm 11.7$ & $77.4 \pm 11.3$ & $<0.0001$ & $65.3 \pm 12.2$ & $65.5 \pm 11.3$ & $64.8 \pm 11.0$ & 0.002 \\
\hline Height (cm) & $175.2 \pm 6.9$ & $172.3 \pm 6.7$ & $170.4 \pm 6.7$ & $<0.0001$ & $161.9 \pm 6.5$ & $159.7 \pm 6.1$ & $157.3 \pm 6.2$ & $<0.0001$ \\
\hline $\mathrm{BMI}\left(\mathrm{kg} / \mathrm{m}^{2}\right)$ & $25.4 \pm 3.4$ & $26.5 \pm 3.5$ & $26.6 \pm 3.4$ & $<0.0001$ & $24.9(4.5)$ & $25.7 \pm 4.3$ & $26.2 \pm 4.3$ & $<0.0001$ \\
\hline $\mathrm{BMI}>30 \mathrm{~kg} / \mathrm{m}^{2}, \%(\mathrm{n})$ & $9.6(6,086)$ & I4.8(1,906) & $16.0(493)$ & $<0.0001$ & $12.9(3,701)$ & $14.8(1,157)$ & $16.7(553)$ & $<0.0001$ \\
\hline Waist circumference $(\mathrm{cm})$ & $88.3 \pm 9.9$ & $93.5 \pm 9.8$ & $95.2 \pm 10.0$ & $<0.0001$ & $77.5 \pm 10.7$ & $81.0 \pm 10.9$ & $83.9 \pm 11.2$ & $<0.000$ I \\
\hline Waist circumference high, \% (n)* & $9.6(6,100)$ & I9.I $(2,468)$ & $25.8(797)$ & $<0.000 \mathrm{I}$ & $15.9(4,564)$ & $23.6(1,839)$ & $33.0(1,096)$ & $<0.000$ I \\
\hline Hypertension, \% (n) & $26.9(17,065)$ & $52.4(6,742)$ & $65.6(2,024)$ & $<0.0001$ & $17.6(5,040)$ & $45.4(3,544)$ & $64.6(2,144)$ & $<0.0001$ \\
\hline Treated hypertension, \% (n) & I.7 (I,088) & $9.2(1,189)$ & $19.6(604)$ & $<0.0001$ & $2.9(828)$ & $12.8(996)$ & $22.94(76 I)$ & $<0.0001$ \\
\hline $\mathrm{SBP}(\mathrm{mmHg})$ & $130.1 \pm 16.4$ & $140.2 \pm 19.6$ & $146.3 \pm 20.9$ & $<0.0001$ & $122.7 \pm 17.3$ & $136.6 \pm 20.4$ & $147.1 \pm 22.2$ & $<0.000$ I \\
\hline $\mathrm{DBP}(\mathrm{mmHg})$ & $77.6 \pm 11.7$ & $84.6 \pm 11.7$ & $84.39 \pm 11.6$ & $<0.0001$ & $74.1 \pm 11.2$ & $79.4 \pm 11.7$ & $80.9 \pm 12.0$ & $<0.0001$ \\
\hline Total cholesterol (mg/dL) & $211.4 \pm 0.2$ & $224.5 \pm 0.4$ & $218.7 \pm 0.73$ & $<0.0001$ & $200.8 \pm 0.24$ & $232.0 \pm 0.5$ & $235.9 \pm 0.7$ & $<0.000$ I \\
\hline LDL cholesterol (mg/dL) & $134.5 \pm 0.1$ & $143.5 \pm 0.3$ & $138.0 \pm 0.7$ & $<0.0001$ & $118 . \pm 0.2$ & $|4| .3 \pm 0.4$ & $143.8 \pm 0.6$ & $<0.000$ I \\
\hline HDL cholesterol (mg/dL) & $55.3 \pm 13.8$ & $57.2 \pm 14.4$ & $58, I \pm 14,6$ & $<0.000$ I & $66.1 \pm 16.5$ & $71.5 \pm 17.6$ & $71.6 \pm 17.5$ & $<0.000$ I \\
\hline Triglycerides (mg/dL) & $108.1 \pm 67.5$ & $118.8 \pm 71.9$ & $113.3 \pm 59.7$ & $<0.0001$ & $79.3 \pm 40.1$ & $96.0 \pm 47.8$ & $102.2 \pm 48.0$ & $<0.0001$ \\
\hline Diabetes, \% (n) & $1.9(1208)$ & $7.1(9 / 8)$ & $11.2(345)$ & $<0.0001$ & I.5 (4I7) & $3.7(292)$ & $5.67(188)$ & $<0.0001$ \\
\hline Treated diabetes, \% (n) & $0.4(255)$ & $2.6(333)$ & $6.6(202)$ & $<0.0001$ & $0.8(230)$ & $2.0(157)$ & $3.7(121)$ & $<0.000$ I \\
\hline Fasting blood glucose (mg/dL) & $96.8 \pm 15.1$ & $103.8 \pm 23.2$ & $106.1 \pm 27.6$ & $<0.0001$ & $90.9 \pm 13.8$ & $97.0 \pm 17.7$ & $99.4 \pm 20.3$ & $<0.000$ I \\
\hline Previous CV event, \% (n) & $0.42(265)$ & $1.65(2 \mid 2)$ & $4.80(148)$ & $<0.0001$ & $0.46(132)$ & $1.36(106)$ & $2.14(71)$ & $<0.0001$ \\
\hline Previous myocardial & $0.13(82)$ & $0.87(I 12)$ & $2.92(90)$ & $<0.0001$ & $0.09(26)$ & $0.21(16)$ & $0.69(23)$ & $<0.0001$ \\
\hline \multicolumn{9}{|l|}{ infarction, \% (n) } \\
\hline Previous stroke, \% (n) & $0.29(184)$ & $0.81(104)$ & $2.01(62)$ & $<0.0001$ & $0.38(109)$ & I.I5 (90) & $\mathrm{I} .48(49)$ & $<0.000$ I \\
\hline EKG changes, \% (n) & $6.2(3,843)$ & $12.0(1,5 \mid 5)$ & $23.0(68 I)$ & $<0.0001$ & $4.7(1,299)$ & $9.8(744)$ & $17.4(560)$ & $<0.0001$ \\
\hline Current smoker, \% (n) & $35.4(22,454)$ & $25.0(3,217)$ & I5.5 (479) & $<0.0001$ & $27.3(7,822)$ & $14.2(1,109)$ & $8,53(283)$ & $<0.000$ I \\
\hline Physical activity, \% (n) & $45.7(28,982)$ & $47.0(6,055)$ & $55.9(1,725)$ & $<0.000 \mathrm{I}$ & $40.6(11,626)$ & $46.7(3,560)$ & $49.2(I, 63 I)$ & $<0.000$ I \\
\hline $\begin{array}{l}\text { High socioprofessional } \\
\text { category, \% (n) }\end{array}$ & $42.4(26,888)$ & $48.6(6,256)$ & $58.0(1,787)$ & $<0.0001$ & I7.5 $(5,008)$ & $24.6(1,916)$ & $25.9(857)$ & $<0.000$ I \\
\hline Total mortality, \% (n) & 0.77 (489) & $2.11(272)$ & $6.26(193)$ & $<0.0001$ & $0.34(97)$ & I.2I (94) & $3.44(1 \mid 4)$ & $<0.0001$ \\
\hline
\end{tabular}

Notes: Comparisons between age groups were performed using ANOVA for quantitative variables and chi-square tests for dichotomous variables; $* \geq 102 \mathrm{~cm}$ in men and $\geq 88 \mathrm{~cm}$ in women.

Abbreviations: ANOVA, analysis of variance; BMI, body mass index; CV, cardiovascular; DBP, diastolic blood pressure; EKG; electrocardiogram; HDL, high-density lipoprotein; LDL, low-density lipoprotein; SBP, systolic blood pressure; SEM, standard error of the mean. 
was less pronounced. The prevalence of hypertension and diabetes also increased with age. The mean values of all lipid parameters were higher in the 55-65 years age group than in the $<55$ years age group. For total cholesterol and LDL cholesterol, the levels increased linearly with age in women. By contrast, in men, total cholesterol and LDL cholesterol decreased among subjects over 65 years of age. HDL cholesterol increased with age, in both men and women.

\section{All-cause mortality associated with obesity in the entire population}

Figure 1 shows all-cause mortality rates (\%) according to WC and BMI categories, in women and men. In both sexes, mortality was higher in participants with a high WC within the three BMI categories $(P<0.001$ for all). In women, for any given WC category, mortality was lower in the higher BMI categories. In men, this was observed for the two lowest categories of WC. So this inverse association was most clearly observed in both men and women with an intermediate WC $(P<0.001)$. Obesity was analyzed as continuous variables using multiple Cox models in the global population,

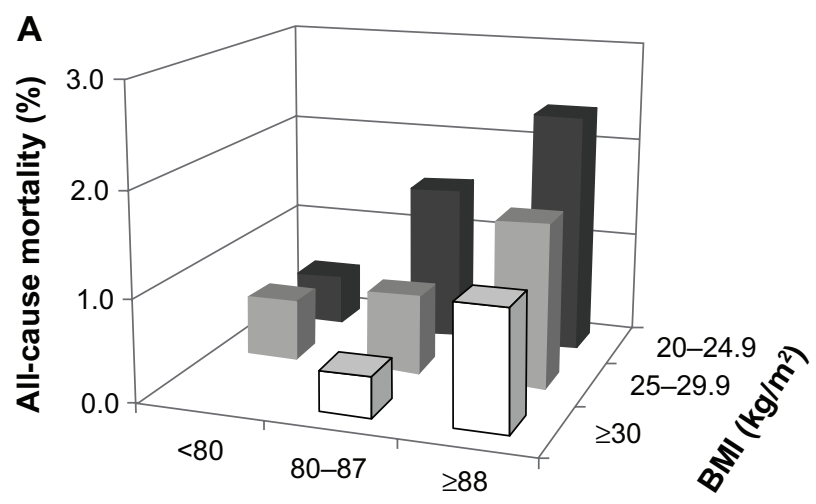

Waist circumference $(\mathrm{cm})$

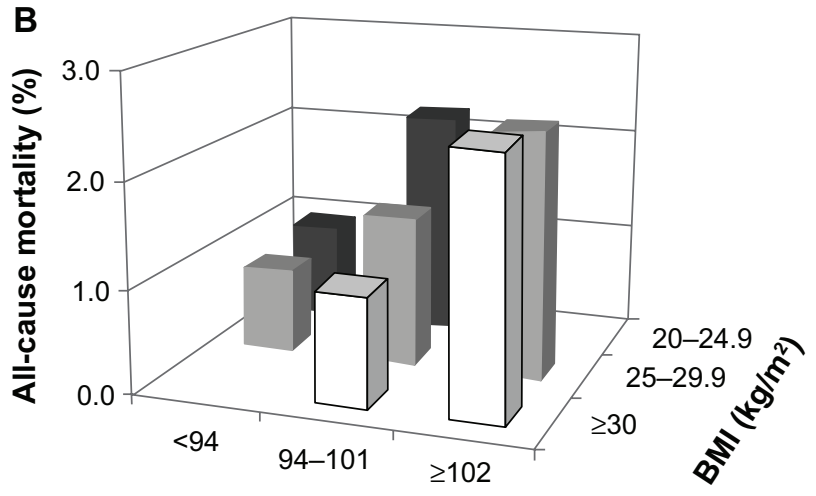

Waist circumference (cm)

Figure I Unadjusted rates for all-cause mortality in (A) women and (B) men, according to $\mathrm{BMI}$ and $\mathrm{WC}$ categories, in the entire study population.

Note: In men with a WC $>102 \mathrm{~cm}$ and a BMI of $20-24.9 \mathrm{~kg} / \mathrm{m}^{2}$, mortality was $8.2 \%$ (4/49 participants) (data not shown).

Abbreviations: BMI, body mass index; WC, waist circumference. which showed that BMI (HR $=1.024$ per BMI unit, $95 \%$ confidence interval [CI]: 1.010-1.040, $P=0.0012$ ) and the WC ( $\mathrm{HR}=1.015$ per centimeter, $95 \% \mathrm{CI}: 1.010-1.021, P<0.0001$ ) were both associated with mortality, after adjustment for age, sex, previous CV events, EKG abnormalities, smoking, socioprofessional category, physical activity, and family history of diabetes and CV disease. However, when BMI and WC were further adjusted for each other, WC adjusted for BMI predicted mortality more strongly $(\mathrm{HR}=1.025$ per centimeter, 95\% CI: 1.016-1.035, $P<0.0001$ ), and BMI adjusted for WC became negatively associated with mortality (HR 0.967 , 95\% CI: $0.943-0.992, P=0.01)$. All these associations were affected very little by further adjustment for hypertension, dyslipidemia, and diabetes (data not shown).

\section{The effect of age on the association between waist circumference, body mass index, and all-cause mortality}

Figure 2 shows all-cause mortality rates (\%) according to WC and BMI categories in individuals $<55$ years, 55-65 years, and $>65$ years. In all age groups, for any given level of WC, BMI was not associated with all-cause mortality $(P>0.10)$. Interestingly, in young ( $<55$ years) subjects, mortality was strongly positively associated with $\mathrm{WC}$, at any given level of BMI (Figure 1A). A similar trend was observed in individuals 55-65 years, though these results were less significant (Figure 1B). In the older group, no such association was observed (Figure 1C). These results were also confirmed in multivariate analyses, shown in Table 2. WC adjusted for BMI was strongly associated with mortality in the two youngest groups. By contrast, this parameter was not associated with mortality in subjects $>65$ years $(P$ interaction $=0.0046)$. Interestingly, age, hypertension, and current smoking were all significantly associated with mortality in subjects $>65$ years, in multivariate analysis. Similar results were obtained when the analysis were carried without excluding those individuals with BMI $<20 \mathrm{~kg} / \mathrm{m}^{2}$ or those who died the first 2 years (data not shown). Finally, we repeated the Cox analysis excluding patients with previous $\mathrm{CV}$ events or with EKG abnormalities. Even in this subpopulation, WC adjusted for BMI was associated with mortality in subjects $<55$ years $(\mathrm{HR}=1.028$, CI: $1.012-1.044, P<0.001)$ and $55-65$ years $(\mathrm{HR}=1.033$, CI: $1.014-1.052, P<0.001)$ but not in subjects $>65$ years (HR $=1.013$, CI: $0.939-1.066, P=\mathrm{NS})$.

\section{Discussion}

The present results demonstrate that $\mathrm{WC}$ was an obesity parameter that was strongly associated with mortality in the 
A

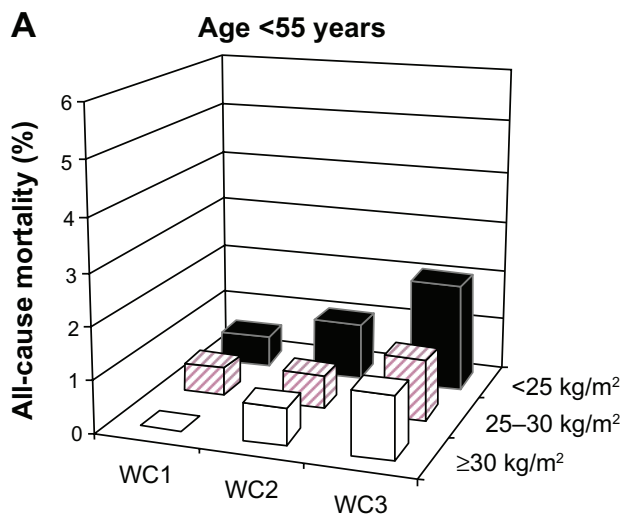

B

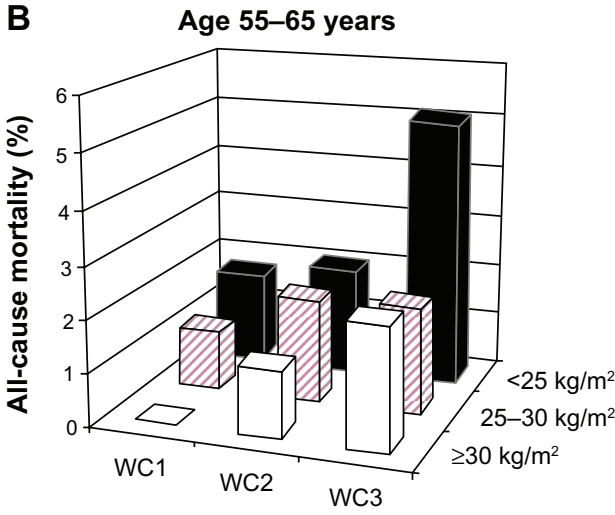

C

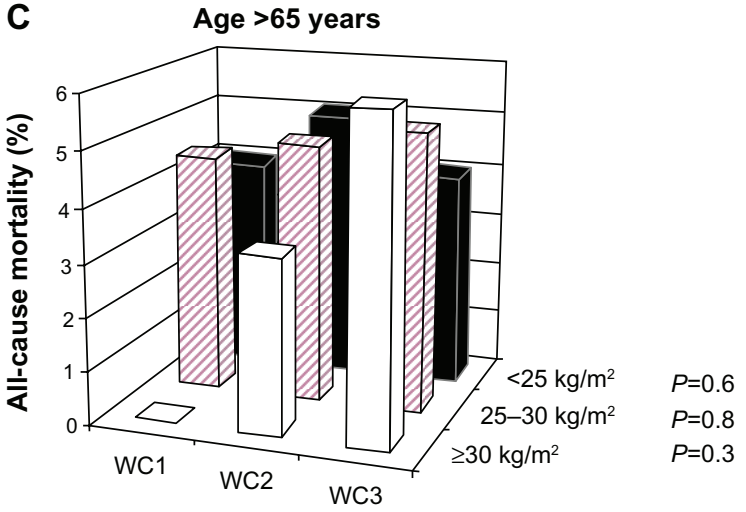

Figure 2 Mortality rates (\%), according to WC and BMI, in individuals $(\mathbf{A})<55$ years, (B) 55-65 years, and (C) $>65$ years.

Notes: WC classification was sex-specified. For men, $\mathrm{WCl}:<94 \mathrm{~cm}$; WC2: 94-101 cm; WC3: $\geq 102 \mathrm{~cm}$. For women, $W C 1:<80 \mathrm{~cm}$; $W C 2: 80-87 \mathrm{~cm}$; $W C 3: \geq 88 \mathrm{~cm}$. $P$-values indicate the effect of WC in each BMI group. Abbreviations: BMI, body mass index; WC, waist circumference.

entire study population, even after adjustment for BMI. This effect was stronger in younger persons and disappeared after the age of 65 years.

WC is widely accepted as a clinical marker of central obesity or visceral fat deposition. ${ }^{4,13}$ The primary result of our study was that WC adjusted for BMI was not associated with mortality in subjects $>65$ years. Conversely, the inverse association between BMI adjusted for WC and mortality was found only in the population $<55$ years. These same conclusions were reached with or without the exclusion of patients with previous $\mathrm{CV}$ events. By contrast, smoking and hypertension remained associated with all-cause mortality in this age group. It is most unlikely that our findings are accounted for by the smaller size of the subpopulation $>65$ years, which represented about 6,000 subjects. Actually, the persistence in this age group, of the associations between mortality and other risk factors, such as smoking and hypertension, argue against this hypothesis. The association between allcause mortality and obesity may also be compounded by reverse causality, whereby low BMI and/or short-term mortality reflect the impact of an underlying disease. However, subjects with a BMI $<20 \mathrm{~kg} / \mathrm{m}^{2}$ and patients with $<2$ years follow-up were excluded from the present analysis to minimize the possible impact of this phenomenon. Contrary to WC, which is a good index of visceral fat deposition, BMI might reflect several components of body composition and not only skeletal fat. It is clearly possible that muscle loss is higher in elderly people than in younger people, without there being any relationship with visceral fat.

There are many putative reasons to explain the lack of association between central obesity and mortality in older subjects. The first hypothesis is linked to a major population effect and is as follows: Obese subjects with higher risk of death cannot reach the age of $>65$ years or have experienced several severe diseases and thus, cannot be observed in our recruited population. However, the prevalence and exposure duration are likely to play an important role. In the present study we noted a markedly higher prevalence of high WC in participants $>65$ years compared with participants 55-65 years. This suggests that a substantial proportion of obese participants $>65$ years have been obese for only a few years. A recent study in young adults showed that the risk of coronary heart disease associated with obesity was strongly amplified if obesity was already present at adolescence, ${ }^{14}$ implying that obesity increases the risk of CV disease only after decades of exposure. Obesity acquired around the age of retirement may have a much weaker impact than if present since early adulthood, as a result of a possibly lifelong poor lifestyle. A short exposure time in a significant proportion of older participants may have "diluted" the prognostic significance of longstanding central obesity.

Our findings corroborate results from several studies that show no association between central obesity and/or metabolic syndrome in older populations. ${ }^{5,6,7,15,16}$ However, they are at apparent odds with previous studies that found a 
Table 2 All-cause mortality associated with obesity and related risk factors, according to age

\begin{tabular}{|c|c|c|c|}
\hline & $<\mathbf{5 5}$ years & $55-65$ years & $>65$ years \\
\hline BMI $\left(\mathrm{kg} / \mathrm{m}^{2}\right)$ & $1.007(0.985-1.030)$ & $1.011(0.983-1.040)$ & $1.030(0.998-1.062)$ \\
\hline $\mathrm{WC}(\mathrm{cm})$ & $1.012(1.004-1.020)^{*}$ & $1.011(1.000-1.021)^{*}$ & $1.012(1.001-1.023)^{*}$ \\
\hline BMI adjusted for WC $\left(\mathrm{kg} / \mathrm{m}^{2}\right)$ & $0.943(0.908-0.979)^{*}$ & $0.960(0.913-1.009)$ & I.007 (0.957-I.059) \\
\hline WC adjusted for $\mathrm{BMI}(\mathrm{cm})$ & $1.030(1.016-1.043)^{* *}$ & $1.023(1.005-1.042)^{*}$ & $1.010(0.993-1.028)$ \\
\hline Age (years) & $1.079(1.066-1.092)^{* *}$ & $1.063(1.023-1.104)^{*}$ & $1.103(1.074-1.133)^{* *}$ \\
\hline Female sex & $0.67 \mid(0.5 \mid 8-0.870)^{*}$ & $0.912(0.664-1.252)$ & $0.720(0.521-0.995)^{*}$ \\
\hline Current smoker (yes/no) & $1.580(1.204-2.072)^{* *}$ & $1.794(1.577-2.042)^{* *}$ & $1.33(1.138-1.555)^{*}$ \\
\hline Hypertension (yes/no) & $1.717(1.442-2.042)^{* *}$ & $1.494(1.196-1.865)^{*}$ & $1.313(1.012-1.705)^{*}$ \\
\hline Total cholesterol (mg/dL) & $0.999(0.997-1.001)$ & $1.000(0.997-1.002)$ & $0.997(0.994-1.001)$ \\
\hline Triglycerides (mg/dL) & $1.000(0.999-1.002)$ & $0.999(0.998-1.001)$ & $1.00 I(0.998-1.003)$ \\
\hline Diabetes (yes/no) & $1.397(0.955-2.042)$ & $1.654(1.172-2.336)^{*}$ & $1.038(0.689-1.565)$ \\
\hline High socioprofessional category (yes/no) & $0.576(0.5 \mathrm{I} I-0.649)^{* * *}$ & $0.874(0.759-1.007)$ & $\mathrm{I} .049(0.859-\mathrm{I} .28 \mathrm{I})$ \\
\hline
\end{tabular}

Notes: Mortality was analyzed using multiple Cox regression models. Data are expressed as hazard ratios ( $95 \%$ confidence intervals). For nonbinary variables, the hazard ratio corresponds to the change in risk per unit (specified in parentheses) of the variable. The model included age, sex, diabetes, hypertension, total cholesterol and triglycerides, current smoking, socioprofessional category, physical activity, and family history of CV disease and diabetes. $* P<0.05$; $* * P<0.000 \mathrm{I}$.

Abbreviations: BMI, body mass index; WC, waist circumference.

significant association between WC (with or without adjustment for BMI) and mortality, even in subjects $>70$ years. ${ }^{3,4,17}$ The prevalence and presumably the duration of exposure to obesity were much lower in our study than those in studies from the USA,, 37 possibly accounting in part for a lower prognostic impact. This explanation cannot account for the differences with the European Prospective Investigation into Cancer and Nutrition (EPIC) study, ${ }^{4}$ in which the prevalence of obesity was apparently quite similar to our study. In this European study, the overall mortality rate was substantially higher, perhaps due in part, to the geographic origin of the study populations, mostly recruited in Northern Europe. ${ }^{18}$ It is quite possible that the higher mortality associated with obesity was related to a higher prevalence and prognostic impact of related risk factors. However, associated risk factors were not assessed in the EPIC study.

Interestingly, hypertension was the conventional risk factor most strongly associated with mortality in participants $>65$ years. The impact of hypertension on mortality may have been underestimated, in the present study, by the effect of antihypertensive treatments. Furthermore, the diagnosis of hypertension was based on clinical measures, which are expected to misclassify many participants due to the role of "white coat" hypertension. The impact of hypertension in older participants could actually be demonstrated only when previous $\mathrm{CV}$ events and EKG abnormalities were excluded from the analysis. It is possible that the association between hypertension and mortality is in part, accounted for by previously accumulated arterial disease or arterial aging. ${ }^{19,20}$ The impact of smoking may have been underestimated by the exclusion of patients with short follow-up or low BMI and by the inclusion of former smokers in the denominator group.
These considerations further emphasize the low relative risk associated with obesity in older subjects in this population. From a physiopathological point of view, visceral fat deposition is deleterious in at least the two following major ways: insulin resistance and inflammation. Our results suggest that in the elderly, these phenomena might have less impact on mortality than other diseases not associated with visceral obesity. Mortality rates are substantially higher in men and women $>65$ years than in younger subjects.

Our study had some limitations. It included very few participants with a BMI $>35 \mathrm{~kg} / \mathrm{m}^{2}(1.54 \%[\mathrm{n}=1220]$ among the men and 3.96\% [n=1576] among the women), therefore, our conclusions do not apply to subjects with severe (class II or class III) obesity. In addition, the population was self-referred on a voluntary basis. Inclusion by self-referral may select health-conscious participants with a low prevalence of associated disorders. For instance, patients with severe diabetes or hypertension may exclude themselves from a primary care checkup as they already benefit from specialized care. However, this study population is seen in a primary care setting, where the identification of modifiable risk factors is clinically most relevant. The follow-up period of 5.6 \pm 2.4 years was rather short, considering the time frame over which obesity exerts its detrimental effects. It is quite possible that obesity-related mortality in older subjects would have been demonstrated with a longer follow-up. However, this limitation cannot account for the decline in obesity-related mortality with aging, nor for the low risk associated with obesity in comparison with other risk factors, such as hypertension and smoking.

In summary, after taking into account associated risk factors, we found that the role of central obesity evaluated by WC adjusted for BMI is significantly associated with 
all-cause mortality, except in the elderly. By contrast, an inverse relationship between obesity evaluated by BMI or BMI adjusted for WC and mortality was observed. These results clearly indicate that $\mathrm{WC}$ and $\mathrm{BMI}$ provide distinct prognostic information. The prognostic significance of central obesity, but not of hypertension or smoking, declines with age. These results do not deny the importance of preventing obesity even in older subjects because obesity clearly remains an important risk factor of functional disabilities ${ }^{21}$ in older subjects. Our data suggest that treatment for hypertension and smoking cessation have a greater potential for decreasing mortality than does weight reduction, especially if obesity is of recent onset.

\section{Acknowledgments}

This study was supported by the Caisse Nationale d'Assurance Maladie des Travailleurs Salariés (CNAMTS, Paris, France) and the Caisse Primaire d'Assurance Maladie de Paris (CPAM-Paris, France).

\section{Disclosure}

The authors report no conflict of interest in this work.

\section{References}

1. Whitlock G, Lewington S, Sherliker P, et al; Prospective Studies Collaboration. Body-mass index and cause-specific mortality in 900000 adults: collaborative analyses of 57 prospective studies. Lancet. 2009;373(9669):1083-1096.

2. Berrington de Gonzalez A, Hartge P, Cerhan JR, et al. Body-mass index and mortality among 1.46 million white adults. $N$ Engl J Med. 2010;363(23):2211-2219.

3. Jacobs EJ, Newton CC, Wang Y, et al. Waist circumference and allcause mortality in a large US cohort. Arch Intern Med. 2010;170(15): 1293-1301.

4. Pischon T, Boeing $\mathrm{H}$, Hoffmann $\mathrm{K}$, et al. General and abdominal adiposity and risk of death in Europe. N Engl J Med. 2008;359(20):2105-2120.

5. Thomas F, Pannier B, Benetos A, Vischer UM. The impact of the metabolic syndrome - but not of hypertension - on all-cause mortality disappears in the elderly. J Hypertens. 2011;29(4):663-668.

6. Mozaffarian D, Kamineni A, Prineas RJ, Siscovick DS. Metabolic syndrome and mortality in older adults: the Cardiovascular Health Study. Arch Intern Med. 2008;168(9):969-978.
7. Sattar N, McConnachie A, Shaper AG, et al. Can metabolic syndrome usefully predict cardiovascular disease and diabetes? Outcome data from two prospective studies. Lancet. 2008;371(9628):1927-1935.

8. Janssen I, Heymsfield SB, Allison DB, Kotler DP, Ross R. Body mass index and waist circumference independently contribute to the prediction of nonabdominal, abdominal subcutaneous, and visceral fat. Am J Clin Nutr. 2002;75(4):683-688.

9. Eckel RH, Alberti KG, Grundy SM, Zimmet PZ. The metabolic syndrome. Lancet. 2010;375(9710):181-183.

10. Lean ME, Han TS, Morrison CE. Waist circumference as a measure for indicating need for weight management. BMJ. 1995;311(6998):158-161.

11. Bigaard J, Tjønneland A, Thomsen BL, Overvad K, Heitmann BL, Sørensen TI. Waist circumference, BMI, smoking, and mortality in middle-aged men and women. Obes Res. 2003;11(7):895-903.

12. Guize L, Thomas F, Pannier B, Bean K, Jego B, Benetos A. All-cause mortality associated with specific combinations of the metabolic syndrome according to recent definitions. Diabetes Care. 2007;30(9): 2381-2387.

13. Bigaard J, Frederiksen K, Tjønneland A, et al. Body fat and fat-free mass and all-cause mortality. Obes Res. 2004;12(7):1042-1049.

14. Tirosh A, Shai I, Afek A, et al. Adolescent BMI trajectory and risk of diabetes versus coronary disease. N Engl J Med. 2011;364(14): 1315-1325.

15. Vyssoulis G, Karpanou E, Adamopoulos D, Tzamou V, Stefanadis C, Vischer UM. Effect of age on interdependence and hierarchy of cardiovascular risk factors in hypertensive patients. Am J Cardiol. 2011;108(2):240-245

16. Salminen M, Kuoppamäki M, Vahlberg T, Räihä I, Irjala K, Kivelä SL. The metabolic syndrome defined by modified International Diabetes Federation criteria and mortality: a 9-year follow-up of the aged in Finland. Diabetes Metab. 2010;36(6 Pt 1):437-442.

17. Zhang C, Rexrode KM, van Dam RM, Li TY, Hu FB. Abdominal obesity and the risk of all-cause, cardiovascular, and cancer mortality: sixteen years of follow-up in US women. Circulation. 2008;117(13): 1658-1667.

18. Müller-Nordhorn J, Binting S, Roll S, Willich SN. An update on regional variation in cardiovascular mortality within Europe. Eur Heart J. 2008;29(10):1316-1326.

19. Benetos A, Salvi P, Lacolley P. Blood pressure regulation during the aging process: the end of the 'hypertension era'? J Hypertens. 2011;29(4):646-652.

20. Vlachopoulos C, Aznaouridis K, Stefanadis C. Prediction of cardiovascular events and all-cause mortality with arterial stiffness: a systematic review and meta-analysis. J Am Coll Cardiol. 2010;55(13): 1318-1327.

21. Al Snih S, Ottenbacher KJ, Markides KS, Kuo YF, Eschbach K, Goodwin JS. The effect of obesity on disability vs mortality in older Americans. Arch Intern Med. 2007;167(8):774-780.
Vascular Health and Risk Management

\section{Publish your work in this journal}

Vascular Health and Risk Management is an international, peerreviewed journal of therapeutics and risk management, focusing on concise rapid reporting of clinical studies on the processes involved in the maintenance of vascular health; the monitoring, prevention and treatment of vascular disease and its sequelae; and the involvement of

\section{Dovepress}

metabolic disorders, particularly diabetes. This journal is indexed on PubMed Central and MedLine. The manuscript management system is completely online and includes a very quick and fair peer-review system, which is all easy to use. Visit http://www.dovepress.com/ testimonials.php to read real quotes from published authors. 